\title{
Criteria of Two-weighted Inequalities for Multidimensional Hardy Type Operator in Weighted Musielak-Orlicz Spaces and Some Application
}

\author{
Rovshan A. Bandaliev \\ Institute of Mathematics and Mechanics, Baku, Azerbaijan \\ Baku, Azerbaijan, Az 1141, B. Vahabzade str., 9 \\ *Corresponding Author: bandaliyev.rovshan@math.ab.az
}

Copyright (C2013 Horizon Research Publishing All rights reserved.

\begin{abstract}
In this paper a two-weight criterion for multidimensional Hardy type operator and its dual operator acting from weighted Lebesgue spaces into weighted Musielak-Orlicz spaces is proved. As application we prove the boundedness of multidimensional geometric mean operator in the weighted Musielak-Orlicz spaces. In particular, from obtained results implies the boundedness of multidimensional Hardy operator and its dual operator acting from usual weighted Lebesgue spaces into weighted variable Lebesgue spaces. In this paper we establish integral-type necessary and sufficient condition on weights, which provides the boundedness of the multidimensional Hardy type operator from weighted Lebesgue spaces into weighted Musielak-Orlicz spaces.
\end{abstract}

Keywords Weights, Weighted Musielak-Orlicz Space, Hardy Type Operator, Multidimensional Geometric Mean Operator, Boundedness

MSC (2000): 46B50, 26D15, 47B38

\section{Introduction}

The notion of Musielak-Orlicz spaces was introduced in [27]. The Musielak-Orlicz spaces are closely connected with modular spaces. It is well known that the first systematic study of modular 
spaces is due to Nakano [29]. Basing on the modular theory Musielak and Orlicz founded in 1959 a theory of Musielak-Orlicz spaces [28]. These spaces have been studied for almost sixty years and there are known a large set of applications of such spaces in various fields of analysis. They were also generalized in many directions, for example, many authors have been considered their generalizations to the vector-valued functions and spaces generated by families of Musielak-Orlicz modulars. Later, a more explicit version of these spaces, namely modular function spaces, were investigated by many mathematicians (see [29]).

For the last forty years a vast amount of research has been carried out on the boundedness of the Hardy operator in classical Lebesgue spaces (see, e.g., [4], [10], [12], [20], [21], [25], [26], [33] and etc). In this paper we establish integral-type necessary and sufficient condition on weights, which provides the boundedness of the multidimensional Hardy type operator from weighted Lebesgue spaces into weighted Musielak-Orlicz spaces. We also investigate the corresponding problems for the dual operator. It is well known that the classical two weight inequality for geometric mean operator closely connected with one-dimensional Hardy inequality (see [12]). Analogously, the Pólya-Knopp type inequalities with multidimensional geometric mean operator is connected with multidimensional Hardy type operator. Therefore in this paper as application of Hardy inequality we prove the boundedness of multidimensional geometric mean operator from weighted Lebesgue spaces into weighted Musielak-Orlicz spaces.

\section{Preliminaries}

Let $R^{n}$ be the $n$-dimensional Euclidean space of points $x=\left(x_{1}, \ldots, x_{n}\right)$ and let $\Omega$ be a Lebesgue measurable subset in $R^{n}$ and $|x|=\left(\sum_{i=1}^{n} x_{i}^{2}\right)^{1 / 2}$. The Lebesgue measure of a set $\Omega$ will be denoted by $|\Omega|$. It is well known that $|B(0,1)|=\frac{\pi^{\frac{n}{2}}}{\Gamma\left(\frac{n}{2}+1\right)}$, where $B(0,1)=\left\{x: x \in R^{n} ;|x|<1\right\}$.

Definition 1. [27, 7] Let $\Omega \subset R^{n}$ be a Lebesgue measurable set. A real function $\varphi: \Omega \times[0, \infty) \mapsto$ $[0, \infty)$ is called a generalized $\varphi$-function if it satisfies:

a) $\varphi(x, \cdot)$ is a $\varphi$-function for all $x \in \Omega$, i.e., $\varphi(x, \cdot):[0, \infty) \mapsto[0, \infty)$ is convex, left-continuous and satisfies $\varphi(x, 0)=0, \lim _{t \rightarrow+0} \varphi(x, t)=0, \lim _{t \rightarrow \infty} \varphi(x, t)=\infty$;

b) $\psi: x \mapsto \varphi(x, t)$ is measurable for all $t \geq 0$.

If $\varphi$ is a generalized $\varphi$-function on $\Omega$, we shortly write $\varphi \in \Phi$. By $L_{0}(\Omega)$ we denote the set of all Lebesgue measurable and everywhere finite real function on $\Omega$.

Definition 2. [22, 7] Let $\varphi \in \Phi$ and be $\rho_{\varphi}$ be define by the expression

$$
\rho_{\varphi}(f):=\int_{\Omega} \varphi(y,|f(y)|) d y \quad \text { for all } \quad f \in L_{0}(\Omega) .
$$

We put $L_{\varphi}=\left\{f \in L_{0}(\Omega): \rho_{\varphi}\left(\lambda_{0} f\right)<\infty \quad\right.$ for some $\left.\lambda_{0}>0\right\}$ and

$$
\|f\|_{L_{\varphi}}=\inf \left\{\lambda>0: \rho_{\varphi}\left(\frac{f}{\lambda}\right) \leq 1\right\} .
$$


The spaces $L_{\varphi}$ is called Musielak-Orlicz space.

Note that the space $L_{\varphi}$ is a Banach function spaces with respect to the norm (1.1) (more detail see [8]). In particular, the Musielak-Orlicz spaces include the classical Lebesgue spaces for $\varphi(x, t)=t^{p}(1 \leq p<\infty)$, the Orlicz spaces for $\varphi(x, t)=\varphi(t)$, and the Lebesgue spaces with variable exponent for $\varphi(x, t)=t^{p(x)}$, where $p: \Omega \mapsto[1, \infty]$ is a Lebesgue measurable functions.

Let $\omega$ be a weight function on $\Omega$, i.e., $\omega$ is a non-negative, almost everywhere positive function on $\Omega$. In this paper we considered the weighted Musielak-Orlicz spaces. We denote

$$
L_{\varphi, \omega}=\left\{f \in L_{0}(\Omega): f \omega \in L_{\varphi}\right\}
$$

It is obvious that the norm in this spaces is given by

$$
\|f\|_{L_{\varphi, \omega}}=\|f \omega\|_{L_{\varphi}} .
$$

By $L_{p, \omega}\left(R^{n}\right)(1 \leq p<\infty)$ we denote the spaces of measurable functions $f$ on $R^{n}$ such that

$$
\|f\|_{L_{p, \omega}\left(R^{n}\right)}=\|f\|_{L_{p, \omega}}=\left(\int_{R^{n}}|f(x) \omega(x)|^{p} d x\right)^{1 / p} .
$$

Definition 3. [27, 7] Let $\varphi \in \Phi$. Then for any $y \in \Omega$ we denote by $\varphi^{*}(y, t)$ the conjugate function of $\varphi(y, t)$, i.e.,

$$
\varphi^{*}(y, u)=\sup _{t \geq 0}(t u-\varphi(y, t))
$$

for all $u \geq 0$ and $y \in \Omega$.

In [27] was proved the following Lemma 1.

Lemma 1. Let $\varphi \in \Phi$. Then

$$
\int_{\Omega}|f(x)||g(x)| d x \leq 2\|f\|_{L_{\varphi}}\|g\|_{L_{\varphi^{*}}}
$$

for all $f \in L_{\varphi}$ and $g \in L_{\varphi^{*}}$, where $L_{\varphi^{*}}$ is a dual spaces of $L_{\varphi}$.

For function $f$ on $\Omega$ we define the functional

$$
\|f\|_{L_{\varphi}}^{1}=\sup _{\|g\|_{L_{\varphi^{*}}} \leq 1} \int_{\Omega} f(x) g(x) d x .
$$

Lemma 2. [27, 7] The expression (1.2) is define the norm on $L_{\varphi}$. This norm is called the Orlicz norm. Moreover, for all $f \in L_{\varphi}$ we have

$$
\|f\|_{L_{\varphi}} \leq\|f\|_{L_{\varphi}}^{1} \leq 2\|f\|_{L_{\varphi}} .
$$

Lemma 3. Let $(x, t) \in \Omega_{1} \times[0, \infty)$, and $\varphi\left(x, t^{1 / p}\right) \in \Phi$ for some $1 \leq p<\infty$. Suppose $f$ : $\Omega_{1} \times \Omega_{2} \mapsto R$. Then the inequality

$$
\|\| f(x, \cdot)\left\|_{L_{p}\left(\Omega_{2}\right)}\right\|_{L_{\varphi}} \leq 2^{1 / p}\|\| f(\cdot, y)\left\|_{L_{\varphi}}\right\|_{L_{p}\left(\Omega_{2}\right)}
$$

is valid. 
Proof. First we prove that from condition $\varphi\left(x, t^{1 / p}\right) \in \Phi$ implies $\varphi(x, t) \in \Phi$, i.e., $L_{\varphi}$ is a Musielak-Orlicz spaces. By the convexity of $\varphi\left(x, t^{1 / p}\right)$ and $\varphi(x, 0)=0$ it follows from the function $\varphi\left(x, t^{1 / p}\right)$ is non-decreasing on $(0, \infty)$. Let $\alpha_{1}+\alpha_{2}=1$, where $\alpha_{1}, \alpha_{2} \geq 0$. It is well known that the function $t^{p}(1 \leq p<\infty)$ is convex at $t \geq 0$. Therefore using the inequality $\left[\alpha_{1} t_{1}+\alpha_{2} t_{2}\right]^{p} \leq \alpha_{1} t_{1}^{p}+\alpha_{2} t_{2}^{p}$ and $(a+b)^{1 / p} \leq a^{1 / p}+b^{1 / p}(a, b \geq 0)$ we have

$$
\begin{gathered}
\varphi\left(x, \alpha_{1} t_{1}+\alpha_{2} t_{2}\right)=\varphi\left(x,\left(\left[\alpha_{1} t_{1}+\alpha_{2} t_{2}\right]^{p}\right)^{1 / p}\right) \leq \\
\leq \varphi\left(x,\left(\alpha_{1} t_{1}^{p}+\alpha_{2} t_{2}^{p}\right)^{1 / p}\right) \leq \alpha_{1} \varphi\left(x, t_{1}\right)+\alpha_{2} \varphi\left(x, t_{2}\right) .
\end{gathered}
$$

All other properties of $\varphi$-function is satisfied automatically. Therefore $\varphi(x, t) \in \Phi$. Further we have

$$
\begin{aligned}
& \|\| f(x, \cdot)\left\|_{L_{p}\left(\Omega_{2}\right)}^{p}\right\|_{L_{\varphi\left(x, t^{1 / p}\right)}}=\inf \left\{\lambda>0: \int_{\Omega_{1}} \varphi\left(x,\left[\frac{\|f(x, \cdot)\|_{L_{p}\left(\Omega_{2}\right)}^{p}}{\lambda}\right]^{1 / p}\right) d x \leq 1\right\}= \\
& \quad=\inf \left\{\mu^{p}>0: \int_{\Omega_{1}} \varphi\left(x, \frac{\|f(x, \cdot)\|_{L_{p}\left(\Omega_{2}\right)}}{\mu}\right) d x \leq 1\right\}=\|\| f(x, \cdot)\left\|_{L_{p}\left(\Omega_{2}\right)}\right\|_{L_{\varphi}}^{p} .
\end{aligned}
$$

Let $g \in L_{\varphi^{*}\left(x, t^{1 / p}\right)}$ and $\|g\|_{L_{\varphi^{*}\left(\cdot, t^{1 / p}\right)}} \leq 1$. Then by Lemma 1 and Lemma 2

$$
\begin{gathered}
\int_{\Omega_{1}}\left(\int_{\Omega_{2}}|f(x, y)|^{p} d y\right) g(x) d x \leq \int_{\Omega_{1}}\left(\int_{\Omega_{2}}|f(x, y)|^{p} d y\right)|g(x)| d x= \\
=\int_{\Omega_{2}}\left(\int_{\Omega_{1}}|f(x, y)|^{p}|g(x)| d x\right) d y \leq 2\|g\|_{L_{\varphi^{*}}\left(\cdot, t^{1 / p}\right)} \int_{\Omega_{2}}\left\|[f(\cdot, y)]^{p}\right\|_{L_{\varphi\left(\cdot, t^{1 / p}\right)}} d y \leq \\
\leq 2 \int_{\Omega_{2}}\left\|[f(\cdot, y)]^{p}\right\|_{L_{\varphi\left(\cdot, t^{1 / p}\right)}} d y=2 \int_{\Omega_{2}}\|f(\cdot, y)\|_{L_{\varphi}}^{p} d y .
\end{gathered}
$$

Hence, $\left\|\int_{\Omega_{2}}|f(\cdot, y)|^{p} d y\right\|_{L_{\varphi\left(\cdot, t^{1 / p}\right)}}^{1} \leq 2 \int_{\Omega_{2}}\|f(\cdot, y)\|_{L_{\varphi}}^{p} d y$. By Lemma 2

$$
\left\|\int_{\Omega_{2}}|f(\cdot, y)|^{p} d y\right\|_{L_{\varphi\left(\cdot, t^{1 / p}\right)}^{1}}^{1} \geq\left\|\int_{\Omega_{2}}|f(\cdot, y)|^{p} d y\right\|_{L_{\varphi\left(\cdot, t^{1 / p}\right)}}=\|\| f(x, \cdot)\left\|_{L_{p}\left(\Omega_{2}\right)}^{p}\right\|_{L_{\varphi\left(x, t^{1 / p}\right)}} .
$$

From equality (1.3) we have

$$
\|\| f(x, \cdot)\left\|_{L_{p}\left(\Omega_{2}\right)}\right\|_{L_{\varphi}} \leq 2^{1 / p}\left(\int_{\Omega_{2}}\|f(\cdot, y)\|_{L_{\varphi}}^{p} d y\right)^{1 / p}=2^{1 / p}\|f(\cdot, y)\|_{L_{\varphi}} \|_{L_{p}\left(\Omega_{2}\right)} .
$$

This proves the Lemma 3. 
Remark 1. Note that in the case $p=1$ Lemma 3 is the analog of generalized Minkowski type inequality in Musielak-Orlicz spaces and was proved in [27]. In the case $\varphi(x, t)=t^{q(x)}$ and $1<p \leq q(x) \leq$ ess $\sup _{x \in R^{n}} q(x)<\infty$ Lemma 3 was proved in [1].

\section{Main Results}

We consider the multidimensional Hardy type operator and its dual operator

$$
H f(x)=\int_{|y|<|x|} f(y) d y \text { and } H^{*} f(x)=\int_{|y|>|x|} f(y) d y
$$

where $f \geq 0$ and $x \in R^{n}$.

Now we prove a two-weight criteria for multidimensional Hardy type operator acting from the weighted Musielak-Orlicz spaces to weighted Lebesgue spaces.

Theorem 1. Let $\varphi\left(x, t^{1 / p}\right) \in \Phi$ for some $1 \leq p<\infty, x \in R^{n}$. Suppose that $v(x)$ and $w(x)$ are weights on $R^{n}$. Then the inequality

$$
\|H f\|_{L_{\varphi, w}} \leq C\|f\|_{L_{p, v}}
$$

holds, for every $f \geq 0$ if and only if there exists $\alpha \in(0,1)$ such that

$$
A(\alpha)=\sup _{t>0}\left(\int_{|y|<t}[v(y)]^{-p^{\prime}} d y\right)^{\frac{\alpha}{p^{\prime}}}\left\|\chi_{\{|z|>t\}}(\cdot)\left(\int_{|y|<|\cdot|}[v(y)]^{-p^{\prime}} d y\right)^{\frac{1-\alpha}{p^{\prime}}}\right\|_{L_{\varphi, w}}<\infty .
$$

Moreover, if $C>0$ is the best possible constant in (2.1) then

$$
\begin{gathered}
\sup _{0<\alpha<1} \frac{p^{\prime} A(\alpha)}{(1-\alpha)\left[\left(\frac{p^{\prime}}{1-\alpha}\right)^{p}+\frac{1}{\alpha(p-1)}\right]^{1 / p}} \leq C \leq \\
\leq 2^{1 / p} \inf _{0<\alpha<1} \frac{A(\alpha)}{(1-\alpha)^{1 / p^{\prime}}} .
\end{gathered}
$$

Proof. Sufficiency. Passing to the polar coordinates, we have

$$
h(y)=\left(\int_{|z|<|y|}[v(z)]^{-p^{\prime}} d z\right)^{\frac{\alpha}{p^{\prime}}}=\left(\int_{0}^{|y|} s^{n-1}\left(\int_{|\xi|=1}[v(s \xi)]^{-p^{\prime}} d \xi\right) d s\right)^{\frac{\alpha}{p^{\prime}}}
$$

where $d \xi$ the surface element on the unit sphere. Obviously, $h(y)=h(|y|)$, i.e., $h(y)$ is a radial function.

Applying Hölder's inequality for $L_{p}\left(R^{n}\right)$ spaces and after some standard transformations, we have

$$
\|H f\|_{L_{\varphi, w}}=\left\|w(\cdot) \int_{|y|<|\cdot|} f(y) d y\right\|_{L_{\varphi}}=
$$




$$
\begin{gathered}
=\left\|w(\cdot) \int_{|y|<|\cdot|}[f(y) h(y) v(y)][h(y) v(y)]^{-1} d y\right\|_{L_{\varphi}} \leq \\
\leq\|w(\cdot)\| f h v\left\|_{L_{p}(|y|<|\cdot|)}\right\|[h v]^{-1}\left\|_{L_{p^{\prime}}(|y|<|\cdot|)}\right\|_{L_{\varphi}}= \\
=\|w(\cdot)\| f h v \chi_{\{|y|<|\cdot|\}}(y)\left\|[h v]^{-1}\right\|_{L_{p^{\prime}}(|y|<|\cdot|)}\left\|_{L_{p}}\right\|_{L_{\varphi}}= \\
=\|\| w(x) f h v \chi_{\{|\cdot|<|x|\}}(\cdot)\left\|[h v]^{-1}\right\|_{L_{p^{\prime}}(|\cdot|<|x|)}\left\|_{L_{p}}\right\|_{L_{\varphi}} .
\end{gathered}
$$

Applying Lemma 3, we have

$$
\begin{gathered}
\|\| w(x) f h v \chi_{\{|\cdot|<|x|\}}(\cdot)\left\|[h v]^{-1}\right\|_{L_{p^{\prime}}(|\cdot|<|x|)}\left\|_{L_{p}}\right\|_{L_{\varphi}} \leq \\
\leq 2^{1 / p}\|\| w(\cdot) f h v \chi_{\{|y|<|\cdot|\}}(y)\left\|[h v]^{-1}\right\|_{L_{p^{\prime}}(|y|<|\cdot|)}\left\|_{L_{\varphi}}\right\|_{L_{p}}= \\
=2^{1 / p}\|f h v\| w(\cdot) \chi_{\{|y|<|\cdot|\}}(y)\left\|[h v]^{-1}\right\|_{L_{p^{\prime}}(|y|<|\cdot|)}\left\|_{L_{\varphi}}\right\|_{L_{p}} .
\end{gathered}
$$

By switching to polar coordinates and after some calculations, we get

$$
\begin{aligned}
& \left\|[h v]^{-1}\right\|_{L_{p^{\prime}}(|y|<|x|)}=\left(\int_{|y|<|x|}[h(|y|) v(y)]^{-p^{\prime}} d y\right)^{1 / p^{\prime}}= \\
& =\left(\int_{0}^{|x|} r^{n-1}[h(r)]^{-p^{\prime}}\left[\int_{|\xi|=1}[v(r \xi)]^{-p^{\prime}} d \xi\right] d r\right)^{1 / p^{\prime}}= \\
& =\left(\int_{0}^{|x|}\left[\int_{0}^{r} s^{n-1}\left(\int_{|\xi|=1}[v(s \xi)]^{-p^{\prime}} d \xi\right) d s\right]^{-\alpha}\left(\int_{|\xi|=1}[v(r \xi)]^{-p^{\prime}} d \xi\right) r^{n-1} d r\right)^{1 / p^{\prime}}= \\
& =\frac{1}{(1-\alpha)^{1 / p^{\prime}}}\left(\int_{0}^{|x|} \frac{d}{d r}\left\{\left(\int_{0}^{r} s^{n-1}\left(\int_{|\xi|=1}[v(s \xi)]^{-p^{\prime}} d \xi\right) d s\right)^{1-\alpha}\right\} d r\right)^{1 / p^{\prime}} \\
& =\frac{1}{(1-\alpha)^{1 / p^{\prime}}}\left(\int_{0}^{|x|} s^{n-1}\left(\int_{|\xi|=1}[v(s \xi)]^{-p^{\prime}} d \xi\right) d s\right)^{\frac{1-\alpha}{p^{\prime}}}= \\
& =\frac{1}{(1-\alpha)^{1 / p^{\prime}}}\left(\int_{|z|<|x|}[v(z)]^{-p^{\prime}} d z\right)^{\frac{1-\alpha}{p^{\prime}}} .
\end{aligned}
$$


Therefore by the condition (2.2), we obtain

$$
\begin{gathered}
\|f h v\| w(\cdot) \chi_{\{|y|<|\cdot|\}}(y)\left\|[h v]^{-1}\right\|_{L_{p^{\prime}}(|y|<|\cdot|)}\left\|_{L_{\varphi}}\right\|_{L_{p}}= \\
=\frac{1}{(1-\alpha)^{1 / p^{\prime}}}\left\|f v\left[h\left\|\chi_{\{|\cdot|>|y|\}} w(\cdot)\left(\int_{|z|<|\cdot|}[v(z)]^{-p^{\prime}} d z\right)^{\frac{1-\alpha}{p^{\prime}}}\right\| \|_{L_{\varphi}}\right]\right\|_{L_{p}} \leq \\
\leq \frac{A(\alpha)}{(1-\alpha)^{1 / p^{\prime}}}\|f v\|_{L_{p}} .
\end{gathered}
$$

Thus

$$
\|H f\|_{L_{\varphi, w}} \leq 2^{1 / p} \frac{A(\alpha)}{(1-\alpha)^{1 / p^{\prime}}}\|f v\|_{L_{p}} \text { for all } \alpha \in(0,1) .
$$

Necessity. Let $f \in L_{p, v}\left(R^{n}\right), f \geq 0$ and the inequality (2.1) is valid. We choose the test function as

$$
f(x)=\frac{p^{\prime}}{1-\alpha}[g(t)]^{-\frac{\alpha}{p^{\prime}}-\frac{1}{p}} v^{-p^{\prime}}(x) \chi_{\{|x|<t\}}(x)+[g(|x|)]^{-\frac{\alpha}{p^{\prime}}-\frac{1}{p}} v^{-p^{\prime}}(x) \chi_{\{|x|>t\}}(x),
$$

where $t>0$ is a fixed number and

$$
g(t)=\int_{|y|<t} v^{-p^{\prime}}(y) d y=\int_{0}^{t} s^{n-1}\left(\int_{|\eta|=1} v^{-p^{\prime}}(s \eta) d \eta\right) d s .
$$

It is obvious that $\frac{d g}{d t}=t^{n-1} \int_{|\eta|=1} v^{-p^{\prime}}(t \eta) d \eta$. Again by switching to polar coordinates from the right hand side of inequality (2.1) we get that

$$
\begin{aligned}
& \|f\|_{L_{p, v}}=\left[\int_{\lfloor x \mid<t}\left(\frac{p^{\prime}}{1-\alpha}\right)^{p}[g(t)]^{-\alpha(p-1)-1} v^{-p^{\prime}}(x) d x+\int_{|x|>t}[g(|x|)]^{-\alpha(p-1)-1} v^{-p^{\prime}}(x) d x\right]^{1 / p}= \\
& =\left[\left(\frac{p^{\prime}}{1-\alpha}\right)^{p}[g(t)]^{\alpha(1-p)}+\int_{t}^{\infty} r^{n-1}[g(r)]^{-\alpha(p-1)-1}\left(\int_{|\xi|=1} v^{-p^{\prime}}(r \xi) d \xi\right) d r\right]^{1 / p}= \\
& =\left[\left(\frac{p^{\prime}}{1-\alpha}\right)^{p}[g(t)]^{\alpha(1-p)}-\frac{1}{\alpha(p-1)} \int_{t}^{\infty} \frac{d}{d r}[g(r)]^{-\alpha(p-1)} d r\right]^{1 / p}= \\
& =\left[\left(\frac{p^{\prime}}{1-\alpha}\right)^{p}[g(t)]^{\alpha(1-p)}+\frac{1}{\alpha(p-1)}\left\{[g(t)]^{-\alpha(p-1)}-\left[\int_{R^{n}} v^{-p^{\prime}}(y) d y\right]^{-\alpha(p-1)}\right\}\right]^{1 / p} \leq \\
& \leq\left[\left(\frac{p^{\prime}}{1-\alpha}\right)^{p}+\frac{1}{\alpha(p-1)}\right]^{1 / p}[g(t)]^{-\frac{\alpha}{p^{\prime}}}=\left[\left(\frac{p^{\prime}}{1-\alpha}\right)^{p}+\frac{1}{\alpha(p-1)}\right]^{1 / p}[h(t)]^{-1} .
\end{aligned}
$$


After some calculations from the left hand side of inequality (2.2), we have

$$
\begin{aligned}
& \|H f\|_{L_{\varphi, w}}=\left\|\int_{|y|<|\cdot|} f(y) d y\right\|_{L_{\varphi, w}} \geq\left\|\chi_{\{|\cdot|>t\}} \int_{|y|<|\cdot|} f(y) d y\right\|_{L_{\varphi, w}}= \\
& =\left\|\chi_{\{|\cdot|>t\}}\left(\frac{p^{\prime}}{1-\alpha} \int_{|y|<t}[g(t)]^{-\frac{\alpha}{p^{\prime}}-\frac{1}{p}} v^{-p^{\prime}}(y) d y+\int_{t<|y|<|\cdot|}[g(|y|)]^{-\frac{\alpha}{p^{\prime}}-\frac{1}{p}} v^{-p^{\prime}}(y) d y\right)\right\|_{L_{\varphi, w}}= \\
& =\left\|\chi_{\{|\cdot|>t\}}\left(\frac{p^{\prime}}{1-\alpha}[g(t)]^{\frac{1-\alpha}{p^{\prime}}}+\int_{t}^{|\cdot|} r^{n-1}[g(r)]^{-\frac{\alpha}{p^{\prime}}-\frac{1}{p}}\left(\int_{\eta \eta \mid=1} v^{-p^{\prime}}(r \eta) d \eta\right) d r\right)\right\|_{L_{\varphi, w}}= \\
& =\left\|\chi_{\{|\cdot|>t\}}\left(\frac{p^{\prime}}{1-\alpha}[g(t)]^{\frac{1-\alpha}{p^{\prime}}}+\frac{\bar{p}^{\prime}}{1-\alpha} \int_{t}^{|\cdot|} \frac{d}{d r}[g(r)]^{\frac{1-\alpha}{p^{\prime}}} d r\right)\right\|_{L_{\varphi, w}}= \\
& \left\|\chi_{\{|\cdot|>t\}}\left[\frac{p^{\prime}}{1-\alpha}[g(t)]^{\frac{1-\alpha}{p^{\prime}}}+\frac{p^{\prime}}{1-\alpha}\left([g(|\cdot|)]^{\frac{1-\alpha}{p^{\prime}}}-[g(t)]^{\frac{1-\alpha}{p^{\prime}}}\right)\right]\right\|_{L_{\varphi, w}}= \\
& =\frac{p^{\prime}}{1-\alpha}\left\|\chi_{\{|\cdot|>t\}}[g(\cdot)]^{\frac{1-\alpha}{p^{\prime}}}\right\|_{L_{\varphi, w}} .
\end{aligned}
$$

Hence, implies that

$$
\begin{aligned}
& \frac{p^{\prime}}{1-\alpha}\left[\left(\frac{p^{\prime}}{1-\alpha}\right)^{p}+\frac{1}{\alpha(p-1)}\right]^{-1 / p}[g(t)]^{\frac{\alpha}{p^{\prime}}}\left\|\chi_{\{|\cdot|>t\}}[g(\cdot)]^{\frac{1-\alpha}{p^{\prime}}}\right\|_{L_{\varphi, w}} \leq C, \\
& \text { i.e., } \frac{p^{\prime} A(\alpha)}{(1-\alpha)\left[\left(\frac{p^{\prime}}{1-\alpha}\right)^{p}+\frac{1}{\alpha(p-1)}\right]^{1 / p}} \leq C \text { for all } \alpha \in(0,1) \text {. }
\end{aligned}
$$

This completes the proof of Theorem 1 .

For the dual operator a theorem below is proved analogously.

Theorem 2. Let $\varphi\left(x, t^{1 / p}\right) \in \Phi$ for some $1 \leq p<\infty, x \in R^{n}$. Suppose that $v(x)$ and $w(x)$ are weights on $R^{n}$. Then the inequality

$$
\left\|H^{*} f\right\|_{L_{\varphi, w}} \leq C\|f\|_{L_{p, v}}
$$

holds, for every $f \geq 0$ if and only if there exists $\gamma \in(0,1)$ such that

$$
B(\gamma)=\sup _{t>0}\left(\int_{|y|>t}[v(y)]^{-p^{\prime}} d y\right)^{\frac{\gamma}{p^{\prime}}}\left\|\chi_{\{|z|<t\}}(\cdot)\left(\int_{|y|>|\cdot|}[v(y)]^{-p^{\prime}} d y\right)^{\frac{1-\gamma}{p^{\prime}}}\right\|_{L_{\varphi, w}}<\infty .
$$

Moreover, if $C>0$ is the best possible constant in (2.3) then

$$
\begin{gathered}
\sup _{0<\gamma<1} \frac{p^{\prime} B(\gamma)}{(1-\gamma)\left[\left(\frac{p^{\prime}}{1-\gamma}\right)^{p}+\frac{1}{\gamma(p-1)}\right]^{1 / p}} \leq C \leq \\
\leq 2^{1 / p} \inf _{0<\gamma<1} \frac{B(\gamma)}{(1-\gamma)^{1 / p^{\prime}}} .
\end{gathered}
$$


Corollary 1. Note that Theorem 1 in the case $n=1, \varphi(x, t)=t^{q}, 1<p \leq q \leq \infty$, for $x \in(0, \infty)$, $\alpha=\frac{s-1}{p-1}$ and $s \in(1, p)$ was proved in [34]. In the case $\varphi(x, t)=t^{q(x)}$ and $1<p \leq q(x) \leq$ ess $\sup _{x \in R^{n}} q(x)<\infty$ Theorem 1 was proved in [3] (see also [2]).

Remark 2. In the case $n=1, \varphi(x, t)=t^{q}, 1<p \leq q \leq \infty$, at $x \in(0, \infty)$, for classical Lebesgue spaces the various variants of Theorem 1 and Theorem 2 were proved in [4], [10], [12], [20], [21], [25], [26], [33] and etc. In particular, in the Lebesgue spaces with variable exponent the boundedness of Hardy type operator was proved in [6], [9], [11], [13], [19], [23], [24] and etc. For Lebesgue spaces with variable exponent the two-weighted criterion for one-dimensional Hardy operator at $x \in[0,1]$ was proved in [19]. Also, other type two-weighted criterion for multidimensional Hardy type operator was proved in [23].

Now we consider the multidimensional geometric mean operator defined as

$$
G f(x)=\exp \left(\frac{1}{|B(0,|x|)|} \int_{B(0,|x|)} \ln f(y) d y\right)
$$

where $f>0$ and $|B(0,|x|)|=|B(0,1)||x|^{n}$. It is obvious that $G\left(f_{1} \cdot f_{2}\right)(x)=$ $G f_{1}(x) \cdot G f_{2}(x)$.

We formulate a two-weight criteria on boundedness of multidimensional geometric mean operator in weighted Musielak-Orlicz spaces.

Theorem 3. Let $\varphi\left(x, t^{1 / p}\right) \in \Phi$ for some $0<p<\infty$ and $x \in R^{n}$. Suppose that $v(x)$ and $w(x)$ are weights functions on $R^{n}$. Then the inequality

$$
\|G f\|_{L_{\varphi, w}} \leq C\|f\|_{L_{p, v}}
$$

holds, if and only if there exists $s \in(1, p)$ such that

$$
D(s)=\sup _{t>0}|B(0, t)|^{\frac{s-1}{p}}\left\|\frac{\chi_{\{|z|>t\}}(\cdot)}{|B(0,|\cdot|)|^{\frac{s}{p}}} \exp \left(\frac{1}{|B(0,|\cdot|)|} \int_{B(0,|\cdot|)} \ln \frac{1}{v(y)} d y\right)\right\|_{L_{\varphi, w}}<\infty .
$$

Moreover, if $C>0$ is the best possible constant in (2.4) then

$$
\sup _{s>1} \frac{e^{\frac{s}{p}}}{\left(e^{s}+\frac{1}{s-1}\right)^{1 / p}} D(s) \leq C \leq 2^{1 / p} \inf _{s>1} e^{\frac{s-1}{p}} D(s) .
$$

Proof. Let $\alpha=\frac{s-1}{p-1}$, where $1<s<p$. We replace $f$ with $f^{\beta}, v$ with $v^{\beta}, w$ with $\frac{w^{\beta}(x)}{|B(0,|x|)|}$, $0<\beta<p$, and $p$ with $\frac{p}{\beta}$ and $\varphi(x, t)$ with $\varphi\left(x, t^{1 / \beta}\right)$ in $(2.1),(2.2)$, we find that for $1<s<\frac{p}{\beta}$

$$
\left\|\frac{w^{\beta}}{|B(0,|\cdot|)|} H\left(f^{\beta}\right)\right\|_{L_{\varphi\left(\cdot, t^{1 / \beta}\right)}}=\left\|\left(\frac{1}{|B(0,|\cdot|)|} \int_{B(0,|\cdot|)} f^{\beta}(y) d y\right)^{1 / \beta}\right\|_{L_{\varphi, w}\left(R^{n}\right)}^{\beta} \leq
$$




$$
\leq C_{\beta}\left(\int_{R^{n}}[f(y) v(y)]^{p} d y\right)^{\beta / p} .
$$

Then the inequality

$$
\left\|\left(\frac{1}{|B(0,|\cdot|)|} \int_{B(0,|\cdot|)} f^{\beta}(y) d y\right)^{1 / \beta}\right\|_{L_{\varphi, w}\left(R^{n}\right)} \leq C_{\beta}^{1 / \beta}\left(\int_{R^{n}}[f(y) v(y)]^{p} d y\right)^{1 / p}
$$

holds if and only if

$$
\begin{gathered}
A\left(\frac{s-1}{p-1}\right)= \\
=\left[\sup _{t>0}\left(\int_{y \mid<t}[v(y)]^{-\frac{\beta p}{p-\beta}} d y\right)^{\frac{s-1}{p} \|}\left\|\left(\frac{\chi_{\{|z|>t\}}(\cdot)}{|B(0,|\cdot|)|^{\frac{p}{p-\beta s}}} \int_{|y|<|\cdot|}[v(y)]^{-\frac{\beta p}{p-\beta}} d y\right)^{\frac{p-\beta s}{\beta p}}\right\| \|_{L_{\varphi, w}}\right]^{\beta}= \\
=B^{\beta}(s, \beta)<\infty
\end{gathered}
$$

and

$$
\sup _{1<s<\frac{p}{\beta}}\left[\frac{\left(\frac{p}{p-s \beta}\right)^{\frac{p}{\beta}}}{\left(\frac{p}{p-s \beta}\right)^{\frac{p}{\beta}}+\frac{1}{s-1}}\right]^{\beta / p} B^{\beta}(s, \beta) \leq C_{\beta} \leq 2^{\frac{\beta}{p}} \inf _{1<s<\frac{p}{\beta}}\left(\frac{p-\beta}{p-s \beta}\right)^{\frac{p-\beta}{p}} B^{\beta}(s, \beta)
$$

By L'Hospital rule, we get

$$
\begin{gathered}
\lim _{\beta \rightarrow+0}\left(\frac{1}{|B(0,|x|)|^{\frac{p}{p-\beta s}}} \int_{|y|<|x|}[v(y)]^{-\frac{\beta p}{p-\beta}} d y\right)^{\frac{p-\beta s}{\beta p}}= \\
=\lim _{\beta \rightarrow+0} \exp \left[\frac{\left[\ln \frac{1}{|B(0,|x|)|}+(p-\beta s) \ln \left(\int_{|y|<|x|}[v(y)]^{-\frac{\beta p}{p-\beta}} d y\right)\right.}{p \beta}\right]= \\
=\lim _{\beta \rightarrow+0} \exp \left[-\frac{s}{p} \ln \left(\int_{|y|<|x|}[v(y)]^{-\frac{\beta p}{p-\beta}} d y\right)+\frac{(p-\beta s)\left(\frac{p}{p-\beta}\right)^{2} \int_{|y|<|x|}[v(y)]^{-\frac{\beta p}{p-\beta}} \ln \frac{1}{v(y)} d y}{p \int_{|y|<|x|}[v(y)]^{-\frac{\beta p}{p-\beta}} d y}\right]=\frac{1}{|B(0,|x|)|^{\frac{s}{p}}} \exp \left(\frac{1}{|B(0,|x|)|} \int_{B(0,|x|)} \ln \frac{1}{v(y)} d y\right) . \\
=\exp \left[\frac{s}{p} \ln \frac{1}{|B(0,|x|)|}+\frac{|y|<|x|}{|B(0,|x|)|} d y\right]
\end{gathered}
$$

Therefore

$$
\begin{gathered}
\lim _{\beta \rightarrow+0} B(s, \beta)= \\
=\sup _{t>0}|B(0, t)|^{\frac{s-1}{p}}\left\|\frac{\chi_{\{|z|>t\}}(\cdot)}{|B(0,|\cdot|)|^{\frac{s}{p}}} \exp \left(\frac{1}{|B(0,|\cdot|)|} \int_{B(0,|\cdot|)} \ln \frac{1}{v(y)} d y\right)\right\|_{L_{\varphi, w}}=
\end{gathered}
$$




$$
=D(s)<\infty
$$

and

$$
\sup _{s>1} \frac{e^{\frac{s}{p}}}{\left(e^{s}+\frac{1}{s-1}\right)^{1 / p}} D(s) \leq \lim _{\beta \rightarrow+0} C_{\beta}^{1 / \beta} \leq 2^{1 / p} \inf _{s>1} e^{\frac{s-1}{p}} D(s) .
$$

Further, we have

$$
\lim _{\beta \rightarrow+0}\left(\frac{1}{|B(0,|x|)|} \int_{B(0,|x|)} f^{\beta}(y) d y\right)^{1 / \beta}=\exp \left(\frac{1}{|B(0,|x|)|} \int_{B(0,|x|)} \ln f(y) d y\right)=G f(x) .
$$

From (2.7) implies that $\lim _{\beta \rightarrow+0} C_{\beta}=1$, and according to (2.5) and (2.8) $\lim _{\beta \rightarrow+0} C_{\beta}^{1 / \beta}=C<\infty$. Therefore the inequality (2.4) is valid. Moreover, from (2.6) for $\beta \rightarrow+0$ we obtain

$$
\|G f\|_{L_{q(\cdot), w}\left(R^{n}\right)} \leq C\|f\|_{L_{p, v}\left(R^{n}\right)}
$$

and by (2.8)

$$
\sup _{s>1} \frac{e^{\frac{s}{p}}}{\left(e^{s}+\frac{1}{s-1}\right)^{1 / p}} D(s) \leq C \leq 2^{1 / p} \inf _{s>1} e^{\frac{s-1}{p}} D(s) .
$$

This completes the proof of Theorem 3 .

Remark 3. Let $\varphi(x, t)=t^{q}$ and $n=1$. Note that the simplest case of (2.4) with $v=w=1$ and $p=q=1$ was considered in [12] and in [18]. Later on, this inequality was generalized in various ways by many authors in [5], [14]-[17], [22], [30]-[32], [34] and etc.

Corollary 2. Let $\varphi(x, t)=t^{q}, 0<p \leq q<\infty$ and let $f$ be a positive function on $R^{n}$. Then

$$
\left(\int_{R^{n}}[G f(x)]^{q}|x|^{\delta q} d x\right)^{1 / q} \leq C\left(\int_{R^{n}} f^{p}(x)|x|^{\mu p} d x\right)^{1 / p}
$$

holds with a finite constant $C$ if and only if

$$
\delta+\frac{n}{q}=\frac{\mu}{n}+\frac{n}{p}
$$

and the best constant $C$ has the following condition:

$$
\sqrt[q]{\frac{p}{n q}} e^{\frac{\mu}{n^{2}}}|B(0,1)|^{\frac{1}{q}-\frac{1}{p}} \sup _{s>1} \frac{e^{\frac{s}{p}}(s-1)^{\frac{1}{p}-\frac{1}{q}}}{\left[(s-1) e^{s}+1\right]^{1 / p}} \leq C \leq \frac{|B(0,1)|^{\frac{1}{q}-\frac{1}{p}} e^{\frac{\mu}{n^{2}}+\frac{1}{q}}}{\sqrt[q]{n}} .
$$

Remark 4. Let $\varphi(x, t)=t^{q}$ and $q=p$. Then the inequality (2.9) is sharp with the constant $C=\frac{e^{\frac{\mu}{n^{2}}+\frac{1}{p}}}{\sqrt[p]{n}}$

\section{Acknowledgements}

The author is supported by the Science Development Foundation under the President of the Republic of Azerbaijan EIF-2010-1(1)-40/06-1. The author would like to express their gratitude to the referee's for his very valuable comments and suggestions. 


\section{REFERENCES}

[1] R.A.Bandaliev, On an inequality in Lebesgue space with mixed norm and with variable summability exponent, Matem. Zametki 84(3), 323-333 (2008) (in Russian): English translation: in Math. Notes 84(3), 303-313 (2008).

[2] R.A.Bandaliev, The boundedness of certain sublinear operator in the weighted variable Lebesgue spaces, Czech. Math. J. 60(2), 327-337 (2010).

[3] R.A.Bandaliev, The boundedness of multidimensional Hardy operator in the weighted variable Lebesgue spaces, Lith. Math. J. 50(3), 249-259 (2010).

[4] J.Bradley, Hardy inequalities with mixed norms, Canadian Math. Bull. 21, 405-408 (1978).

[5] J.A.Cochran and C.-S.Lee, Inequalities related to Hardy's and Heinig's, Math. Proc. Camb. Phil.Soc. 96, 1-7 (1984).

[6] D.Cruz-Uribe, A.Fiorenza and C.J.Neugebauer, The maximal function on variable $L^{p}$ spaces, Ann. Acad. Sci. Fenn. Math. 28(1), 223-238 (2003).

[7] L.Diening, Lebesgue and Sobolev spaces with variable exponent, Habilitation thesis, Germany, Freiburg, 2007.

[8] L.Diening, P.Harjulehto, P.Hästö, and M. Rǔžička, Lebesgue and Sobolev spaces with variable exponents, Springer Lecture Notes, v.2017, Springer-Verlag, Berlin, 2011.

[9] L.Diening and S.Samko, Hardy inequality in variable exponent Lebesgue spaces, Frac. Calc. Appl. Anal. 10(1), 1-18 (2007) .

[10] V.Kokilashvili, On Hardy's inequality in weighted spaces, Bull. Acad. Sci. Georgian SSR 96(1), 37-40 (1978) (in Russian).

[11] D.E.Edmunds, V.Kokilashvili and A.Meskhi, On the boundedness and compactness of weighted Hardy operators in spaces $L^{p(x)}$, Georgian Math.J. 12(1), 27-44 (2005).

[12] G.H.Hardy, J.E. Littlewood, and G.Pólya, Inequalities, Cambridge University Press, 1934.

[13] P.Harjulehto, P.Hästö and M.Koskenoja, Hardy's inequality in a variable exponent Sobolev space, Georgian Math.J. 12(3), 431-442 (2005).

[14] H.P.Heinig, Some extensions of inequalities, SIAM J. Math. Anal. 6, 698-713 (1975) .

[15] H.P. Heinig, Weighted inequalities in Fourier analysis, in: "Nonlinear Analysis, Function spaces and Applications" 4 Proceedings of the Spring School at Roudnice nad Labem, 1990 Teubner Texte 119, Leipzig, 1990, 42-85. (6)(1975) 698-713.

[16] P.Jain, L.E.Persson, and A.Wedestig, From Hardy to Carleman and general mean-type inequalities, Function Spaces and Appl. 117-130 (2000).

[17] P.Jain, L.E.Persson, and A.Wedestig, Carleman-Knopp type inequalities via Hardy's inequality, Math. Inequal. Appl. 4(3), 343-355 (2001).

[18] K.Knopp, Über reihen mit positivern gliedern, J. London Math. Soc. 3, 205-211 (1928). 
[19] T.S.Kopaliani, On some structural properties of Banach function spaces and boundedness of certain integral operators, Czech. Math. J. 54(129), 791-805 (2004).

[20] M.Krbec, B.Opic, L.Pick and J.Rákosník, Some recent results on Hardy type operators in weighted function spaces and related topics, in: "Function Spaces, Differential Operators and Nonlinear Analysis", 158-184 (1993), Teubner, Stuttgart.

[21] A.Kufner and L.E.Persson, Integral inequalities with weights, (World Scientific Publishers, Singapore, 2002).

[22] E.R. Love, Inequalities related to those of Hardy and of Cochran and Lee, Math. Proc. Camb. Phil.Soc. 99(1986) 395-408.

[23] F.I.Mamedov and A.Harman, On a weighted inequality of Hardy type in spaces $L^{p(\cdot)}$, J. Math. Anal. Appl. 353(2), 521-530 (2009).

[24] R.A.Mashiyev, B.Çekiç, F.I.Mamedov and S.Ogras, Hardy's inequality in power-type weighted $L^{p(\cdot)}(0, \infty)$ spaces. J. Math. Anal. Appl. 334(1), 289-298 (2007).

[25] V.G.Maz'ya, Sobolev spaces, (Springer-Verlag, Berlin, 1985).

[26] B.Muckenhoupt, Hardy's inequality with weights, Studia Math. 44, 31-38 (1972).

[27] J.Musielak, Orlicz spaces and modular spaces, Lecture Notes in Math. vol.1034, (Springer-Verlag, Berlin-Heidelberg-New York, 1983).

[28] J.Musielak and W.Orlicz, On modular spaces, Studia Math. 18, 49-65 (1959).

[29] H.Nakano, Modulared semi-ordered linear spaces, Maruzen, Co., Ltd., Tokyo (1950).

[30] B.Opic, P.Gurka, Weighted inequalities for geometric means, Proc. Amer. Math. Soc. 120(3), 771-779 (1994).

[31] L.-E. Persson, V.D. Stepanov, Weighted integral inequalities with the geometric mean operator, J. Inequal. Appl. 7(5), 727-746 (2002).

[32] L.Pick, B.Opic, On geometric mean operator, J. Math. Anal. Appl. 183(3), 652-662 (1994).

[33] G.Talenti, A class of inequalities, Boll.Un.Mat.Ital. 2, 622-631 (1969.

[34] A. Wedestig, Some new Hardy type inequalities and their limiting inequalities, J.of Ineq. in Pure and Appl. Math. 4, art. 61(3) (2003). 